\title{
THE UNIONS OF TRA JECTORIAL SERIES OF LINEAL ELEMENTS GENERATED BY THE PLANE MOTION OF A RIGID BODY
}

\author{
BY \\ DON MITTLEMAN
}

1. The motion of a plane, rigid, macroscopic body, moving freely in a plane under the action of any force which depends solely on the position of the body, may be described by the motion of a lineal element composed of the center of gravity and a principal axis of inertia. Of the coordinates $(x, y, \theta)$ of the lineal element $E,(x, y)$ denotes also the position of the center of gravity of the body and $\theta$ the angle between a principal axis of inertia and the $x$-axis. The equations of motion of $E$ are

$$
M \ddot{x}=X(x, y, \theta), \quad M \ddot{y}=Y(x, y, \theta), \quad I \ddot{\theta}=T(x, y, \theta),
$$

where $M$ is the mass, $I$ the moment of inertia of the body about the center of gravity, $X$ and $Y$ the resultant forces in the $x$ and $y$ directions respectively, and $T$ the torque. Dots will everywhere indicate differentiation with respect to the time $t$.

At any initial time, say $t=0$, the body may be launched from any configuration $\left(x_{0}, y_{0}, \theta_{0}\right)$, with any linear velocity $\left(\dot{x}_{0}, \dot{y}_{0}\right)$, and with any angular velocity $\dot{\theta}_{0}$. The lineal element $E$ generates a unique trajectorial series. By varying the arbitrary constants $x_{0}, y_{0}, \theta_{0}, \dot{x}_{0}, \dot{y}_{0}, \dot{\theta}_{0}$, there is obtained in all, since each trajectorial series may be described from any one of its elements, $\infty^{5}$ trajectorial series. Thus, to each field of force and each macroscopic body there.corresponds a definite quintuply infinite system of series.

2. Let the maximum diameter $r$ of the macroscopic body approach zero in such a way that the position of the center of gravity with respect to the body, the directions of the principal axes of inertia with respect to the body, and the relative magnitude of the two principal moments of inertia remain constant. The resulting ideal system is called an element-particle and its equations of motion are defined as

$$
\ddot{x}=\lim _{r \rightarrow 0} X / M, \quad \ddot{y}=\lim _{r \rightarrow 0} Y / M, \quad \ddot{\theta}=\lim _{r \rightarrow 0} T / I .
$$

As for the macroscopic body, to each field of force and element-particle there corresponds a definite quintuply infinite system of trajectorial series, which system would be found by eliminating the time from the equations of motion.

3. The coordinates $(x, y, \theta)$ in equations (1) are lagrangian coordinates.

Presented to the Society, April 17, 1948; received by the editors October 1, 1947. 
Thus, when the acceleration field, whose $x$ and $y$ components are denoted by $\phi(x, y)$ and $\psi(x, y)$ respectively, is a potential field, the functions $X, Y$, and $T$ are derivable from a potential function $U(x, y, \theta)$. As this property is not true for the limit functions which are the right-hand members of (2), it was desirable to introduce the concept of microscopic body, for which the precise definition and the equations of motion are given in $\S 8$. The components of the force and the torque acting on a microscopic body have the desired property: if there exists a function $u(x, y)$ such that $u_{x}=\phi$ and $u_{y}=\psi$, then there exists a function $U(x, y, \theta)$ such that $U_{x}=X, U_{y}=Y, U_{\theta}=T$, where now $X$ and $Y$ are the $x$ and $y$ components of the total force and $T$ is the torque acting on the microscopic body. The subscripts denote partial derivatives.

For the microscopic body, as for the macroscopic body and the elementparticle, there corresponds to each field of force and each microscopic body $\infty^{s}$ trajectorial series.

4. In each of the three cases described there exist, as subsets of the trajectorial series, trajectorial unions. Union is used in the ordinary sense as a series whose elements coincide in direction with the tangents to the base curve of the series; a trajectorial union is a trajectorial series which is a union. It is the purpose of this paper to investigate in detail the geometric character of trajectorial unions for the general element-particle $\left.{ }^{1}\right)$.

\section{The EQUATIONS OF MOTION}

5. If $\phi$ and $\psi\left({ }^{2}\right)$ are the $x$ and $y$ components of the acceleration field $F$, and if $\rho(\alpha, \beta)$ is the density function for the macroscopic body $R$ referred to the principal axes of inertia of the body, then the equations of motion are given by (1) where explicitly

$$
\begin{array}{rlrl}
M & =\iint_{R} \rho d A, & I=\iint_{R} \rho\left(\alpha^{2}+\beta^{2}\right) d A, \\
X & =\iint_{R} \rho \phi\left(x+\alpha^{\prime}, y+\beta^{\prime}\right) d A, & Y=\iint_{R} \rho \psi\left(x+\alpha^{\prime}, y+\beta^{\prime}\right) d A, \\
T & =\iint_{R} \rho \psi\left(x+\alpha^{\prime}, y+\beta^{\prime}\right) \alpha^{\prime} d A-\iint_{R} \rho \phi\left(x+\alpha^{\prime}, y+\beta^{\prime}\right) \beta^{\prime} d A, \\
\alpha^{\prime} & =\alpha \cos \theta-\beta \sin \theta, \quad \beta^{\prime}=\alpha \sin \theta+\beta \cos \theta .
\end{array}
$$

It may be easily verified that if the acceleration field $F$ is derivable from a potential $u$, that is, if $\phi=u_{x}$ and $\psi=u_{y}$, then there exists a potential function

(1) The geometric characterization of the unions associated with a macroscopic body, with a microscopic body and the trajectorial series in all three cases will be treated in later papers. The corresponding theories in space, on a surface, and so on, will also be reserved for future papers.

(2) The functions $\phi$ and $\psi$ are assumed to be uniform and to possess continuous partial derivatives of the third order in the region of the plane considered. 
$U$ such that $X=U_{x}, Y=U_{y}$, and $T=U_{\theta}$. In particular,

$$
U=\iint_{R} \rho u\left(x+\alpha^{\prime}, y+\beta^{\prime}\right) d A .
$$

Those bodies for which every direction from the center of gravity of the body may serve as a principal axis of inertia will be called radially isotropic. It is self-evident that the components of the force and the torque acting on a radially isotropic body are independent of $\theta$.

6. For the element-particle, equations (2) reduce to

(4.1) $\ddot{x}=\phi(x, y)$,

(4.2) $\ddot{y}=\psi(x, y)$,

(4.3) $\ddot{\theta}=2^{-1}\left(\psi_{x}-\phi_{y}\right)+2^{-1}\left(I_{\alpha}^{*}-I_{\beta}^{*}\right)\left[\left(\psi_{x}+\phi_{y}\right) \cos 2 \theta+\left(\psi_{y}-\phi_{x}\right) \sin 2 \theta\right]$,

where

$$
I_{\alpha}^{*}=\lim _{r \rightarrow 0} \frac{\iint_{R} \rho \alpha^{2} d A}{I}, \quad I_{\beta}^{*}=\lim _{r \rightarrow 0} \frac{\iint_{R} \rho \beta^{2} d A}{I}, \quad I_{\alpha}^{*}+I_{\beta}^{*}=1
$$

and where the limiting process conforms with the conditions of $\$ 2$.

The $\lim _{r \rightarrow 0} T / I$ was determined as follows. The function $\phi\left(x+\alpha^{\prime}, y+\beta^{\prime}\right)$, considered as a function of $\alpha$ and $\beta$, has the expansion about the origin

$$
\begin{aligned}
\phi\left(x+\alpha^{\prime}, y+\beta^{\prime}\right)=\phi & +\alpha\left(\cos \theta \frac{\partial}{\partial x}+\sin \theta \frac{\partial}{\partial y}\right) \phi \\
& +\beta\left(-\sin \theta \frac{\partial}{\partial x}+\cos \theta \frac{\partial}{\partial y}\right)_{\phi} \\
& +\frac{\alpha^{2}}{2 !}\left(\cos \theta \frac{\partial}{\partial x}+\sin \theta \frac{\partial}{\partial y}\right)^{2} \phi \\
& +\alpha \beta\left(\cos \theta \frac{\partial}{\partial x}+\sin \theta \frac{\partial}{\partial y}\right) \\
& \cdot\left(-\sin \theta \frac{\partial}{\partial x}+\cos \theta \frac{\partial}{\partial y}\right)_{\phi} \\
& +\frac{\beta^{2}}{2 !}\left(-\sin \theta \frac{\partial}{\partial x}+\cos \theta \frac{\partial}{\partial y}\right)^{2} \phi
\end{aligned}
$$

where $\phi=\phi(x, y), \quad \phi_{x}=\phi_{x}(x, y), \quad \phi_{y}=\phi_{y}(x, y), \quad \phi_{x x}=\phi_{x x}\left(x+\tau \alpha^{\prime}, \quad y+\tau \beta^{\prime}\right)$, $\phi_{x y}=\phi_{x y}\left(x+\tau \alpha^{\prime}, y+\tau \beta^{\prime}\right), \phi_{y y}=\phi_{y y}\left(x+\tau \alpha^{\prime}, y+\tau \beta^{\prime}\right)$, and where $0<\tau<1$. A similar expansion can be obtained for $\psi\left(x+\alpha^{\prime}, y+\beta^{\prime}\right)$. Substituting these expansions into $T$, as given in (3), noting that the $\alpha, \beta$ axes coincide with the principal axes of inertia of the body and that the second order partial deriva- 
tives of $\phi$ and $\psi$ are continuous, the $\lim _{r \rightarrow 0} T / I$ is seen to be as given in the right side of equation (4.3).

The right sides of (4.1) and (4.2) were determined in similar fashion. However, in these cases it was necessary to expand the functions $\phi$ and $\psi$ to include only first order partial derivatives.

7. In general, if the functions $\phi$ and $\psi$ are the components of a potential field of force and $u(x, y)$ is the potential function, there will not exist a potential function $U(x, y, \theta)$ such that the right members of (4) are $U_{x}, U_{y}$, and $U_{\theta}$. However, there are two exceptional cases when $U$ will exist. For any elementparticle, if

$$
u(x, y)=\left(c_{1}+c_{2}\right) x^{2}+c_{3} x y+c_{1} y^{2}+c_{4} x+c_{5} y
$$

where the $c_{i}$ are constants, then

$$
U(x, y, \theta)=u(x, y)+2^{-1}\left(I_{\alpha}^{*}-I_{\beta}^{*}\right)\left(c_{3} \sin 2 \theta+c_{2} \cos 2 \theta\right) .
$$

For the special element-particle for which $I_{\alpha}^{*}=I_{\beta}^{*}$ (which will be called a symmetric element-particle) the torque is identically zero $\left.{ }^{(3}\right)$ and, therefore, we may set $U(x, y, \theta)=u(x, y)$. These are the only exceptional cases.

8. The equations of motion for the microscopic body are determined as follows. The functions $\phi\left(x+\alpha^{\prime}, y+\beta^{\prime}\right)$ and $\psi\left(x+\alpha^{\prime}, y+\beta^{\prime}\right)$ may be expanded in the same manner as was done in (5) but this time to include the third order partial derivatives. These expansions are to be substituted into the integrals for $X$ and $Y$ of (3). In the integral for $T$ of (3), the expansion (5) for $\phi\left(x+\alpha^{\prime}\right.$, $\left.y+\beta^{\prime}\right)$ and the similar expansion for $\psi\left(x+\alpha^{\prime}, y+\beta^{\prime}\right)$ may be used. The expansion for $X$ becomes

$X=M \phi+4^{-1} I \nabla^{2} \phi+4^{-1}\left(I_{\alpha}-I_{\beta}\right)\left[\left(\phi_{x x}-\phi_{y y}\right) \cos 2 \theta+2 \phi_{x y} \sin 2 \theta\right]+Q_{1}$,

where

$$
Q_{1}=\frac{1}{3 !} \iint_{R} \rho\left(\alpha^{\prime} \frac{\partial}{\partial x}+\beta^{\prime} \frac{\partial}{\partial y}\right)^{3} \phi d A,
$$

where $\nabla^{2}$ is the laplacian operator, where $\phi$ and its second order partial dederivatives are functions of $x$ and $y$ but where the variables which appear in the third order derivatives of $\phi$ are $x+\tau_{1} \alpha^{\prime}$ and $y+\tau_{1} \beta^{\prime}$, with $0<\tau_{1}<1$. $I_{\alpha}$ and $I_{\beta}$ are the moments of inertia about the $\beta$ and $\alpha$ axes respectively, that is,

$$
I_{\alpha}=\iint_{R} \rho \alpha^{2} d A, \quad I_{\beta}=\iint_{R} \rho \beta^{2} d A .
$$

Let $q_{1}$ be the largest of the four numbers which are the maxima of $\left|\phi_{x x x}\right|, \cdots$,

(3) This is a special case of the more general theorem that in a potential field the angular velocity of a macroscopic, radially isotropic body is constant. 
$\left|\phi_{y y y}\right|$ considered as functions of $\alpha, \beta$. Then, if $r$ is the maximum diameter of $R$,

$$
\begin{aligned}
\left|Q_{1}\right| \leqq & \frac{1}{3 !} \iint_{R} \rho\left|\left(\alpha^{\prime} \frac{\partial}{\partial x}+\beta^{\prime} \frac{\partial}{\partial y}\right)^{3} \phi\right| d A \leqq \frac{q_{1}}{3 !} \iint_{R} \rho\left\{\left|\alpha^{\prime}\right|+\left|\beta^{\prime}\right|\right\}^{3} d A \\
& \leqq \frac{8 q_{1}}{3 !} \iint_{R} \rho\{|\alpha|+|\beta|\}^{3} d A \leqq \frac{4 q_{1}}{3}(8 I r)=\frac{32}{3} q_{1} \text { Ir. }
\end{aligned}
$$

In an analagous manner the expansion for $Y$ is obtained, the function $Q_{2}$ defined, and the number $q_{2}$ determined.

For $T$ the expansion becomes

$T=2^{-1} I\left(\psi_{x}-\phi_{y}\right)+2^{-1}\left(I_{\alpha}-I_{\beta}\right)\left[\left(\psi_{x}+\phi_{y}\right) \cos 2 \theta+\left(\psi_{y}-\phi_{x}\right) \sin 2 \theta\right]+Q_{3}$ where

$$
Q_{3}=2^{-1} \iint_{R} \rho\left(\alpha^{\prime} \frac{\partial}{\partial x}+\beta^{\prime} \frac{\partial}{\partial y}\right)^{2}\left(\psi \alpha^{\prime}-\phi \beta^{\prime}\right) d A,
$$

where the first order derivatives of $\phi$ and $\psi$ are functions of $x$ and $y$ but where the variables which appear in the second order derivatives are $x+\tau_{3} \alpha^{\prime}$ and $y+\tau_{3} \beta^{\prime}$, with $0<\tau_{3}<1$. Let $q_{3}$ be the largest of the six numbers which are the maxima of $\left|\phi_{x x}\right|, \cdots,\left|\psi_{y y}\right|$ considered as functions of $\alpha, \beta$. Then,

$$
\begin{aligned}
\left|Q_{3}\right| & \leqq 2^{-1} \iint_{R} \rho\left|\left(\alpha^{\prime} \frac{\partial}{\partial x}+\beta^{\prime} \frac{\partial}{\partial y}\right)^{2}\left(\psi \alpha^{\prime}-\phi \beta^{\prime}\right)\right| d A \\
& \leqq \frac{q_{3}}{2} \iint_{R} \rho\left\{\left|\alpha^{\prime}\right|+\left|\beta^{\prime}\right|\right\}^{3} d A \\
& \leqq 4 q_{3} \iint_{R} \rho\{|\alpha|+|\beta|\}^{3} d A \\
& \leqq 32 q_{3} I r .
\end{aligned}
$$

The bounds $q_{1}, q_{2}$, and $q_{3}$ are functions of $x$ and $y$. In those regions of the $x, y$ plane for which the $q_{i}^{\prime}$ 's are bounded, we have, for every macroscopic body,

$$
\begin{aligned}
\left|X-M \phi-4^{-1} I \nabla^{2} \phi-4^{-1}\left(I_{\alpha}-I_{\beta}\right)\left[\left(\phi_{x x}-\phi_{y y}\right) \cos 2 \theta+2 \phi_{x y} \sin 2 \theta\right]\right| \\
=\left|Q_{1}\right| \leqq \frac{32}{3} q_{1} I r
\end{aligned}
$$

and two similar expressions for $Y$ and $T$.

A body shall be called microscopic when its maximum diameter is so small that each of the three integrals $Q_{1}, Q_{2}$, and $Q_{3}$ may be neglected in the computation of the forces $X$ and $Y$ and the torque $T$ acting on the body. The 
purpose of this definition is to introduce a system in which the torque and the components of the force approximate the comparable quantities acting on an element-particle, but which system has the added property that if the body is in a potential field, then the torque and the components of the total force acting on it are also derivable from a potential function.

The equations of motion for a microscopic body are

$$
\begin{aligned}
M \ddot{x}=X= & M \phi+4^{-1} I \nabla^{2} \phi \\
& +4^{-1}\left(I_{\alpha}-I_{\beta}\right)\left[\left(\phi_{x x}-\phi_{y y}\right) \cos 2 \theta+2 \phi_{x y} \sin 2 \theta\right] \\
M \ddot{y}=Y= & M \psi+4^{-1} I \nabla^{2} \psi \\
& +4^{-1}\left(I_{\alpha}-I_{\beta}\right)\left[\left(\psi_{x x}-\psi_{y y}\right) \cos 2 \theta+2 \psi_{x y} \sin 2 \theta\right] \\
I \ddot{\theta}=T= & 2^{-1} I\left(\psi_{x}-\phi_{y}\right) \\
& +2^{-1}\left(I_{\alpha}-I_{\beta}\right)\left[\left(\psi_{x}+\phi_{y}\right) \cos 2 \theta+\left(\psi_{y}-\phi_{x}\right) \sin 2 \theta\right] .
\end{aligned}
$$

9. TheOREM 1. For a microscopic body, if $\phi(x, y)$ and $\psi(x, y)$ are derivable from a potential $(x, y)$, then $X, Y$, and $T$ are derivable from the potential function

$U(x, y, \theta)=M u+4^{-1} I \nabla^{2} u+4^{-1}\left(I_{\alpha}-I_{\beta}\right)\left[\left(u_{x x}-u_{y y}\right) \cos 2 \theta+2 u_{x y} \sin 2 \theta\right]$, and conversely.

The first part of the theorem is obvious. To establish the converse, note that the conditions $X_{\theta}-T_{x}=0, Y_{\theta}-T_{y}=0$, and not both $I_{\alpha}$ and $I_{\beta}$ equal to zero imply that $\phi_{y}-\psi_{x}=$ constant. Then $X_{y}-Y_{x}=0$ implies the constant is zero. Therefore the potential exists.

\section{THE DIFFERENTIAL EQUATIONS OF THE TRAJECTORIAL SERIES}

10. To obtain the differential equations of the trajectorial series associated with the equations

$$
\ddot{x}=X(x, y, \theta), \quad \ddot{y}=Y(x, y, \theta), \quad \ddot{\theta}=T(x, y, \theta),
$$

it is necessary to eliminate the time. Along any series, $y$ and $\theta$ are functions of $x$. If accents are used to indicate differentiation with respect to $x$, then there exist the following relations between $y(x)$ and $\theta(x)$ on the one hand and $x(t), y(t)$, and $\theta(t)$ on the other.

$$
\begin{array}{rlrl}
\frac{d y}{d t} & =y^{\prime} \frac{d x}{d t}, & \frac{d \theta}{d t} & =\theta^{\prime} \frac{d x}{d t} \\
\frac{d^{2} y}{d t^{2}}=y^{\prime \prime}\left(\frac{d x}{d t}\right)^{2}+y^{\prime} \frac{d^{2} x}{d t^{2}}, & \frac{d^{2} \theta}{d t^{2}}=\theta^{\prime \prime}\left(\frac{d x}{d t}\right)^{2}+\theta^{\prime} \frac{d^{2} x}{d t^{2}} .
\end{array}
$$

The elimination of the time derivatives between the equations (7) and (8) results in one time-independent equation 


$$
\frac{Y-y^{\prime} X}{y^{\prime \prime}}=\frac{T-\theta^{\prime} X}{6^{\prime \prime}} \text {. }
$$

Differentiate (7) with respect to the time and eliminate all time derivatives between these three new equations, (7), (8), and

$$
\begin{aligned}
& \frac{d^{3} y}{d t^{3}}=y^{\prime \prime \prime}\left(\frac{d x}{d t}\right)^{3}+3 y^{\prime \prime} \frac{d x}{d t} \frac{d^{2} x}{d t^{2}}+y^{\prime} \frac{d^{3} x}{d t^{3}} \\
& \frac{d^{3} \theta}{d t^{3}}=\theta^{\prime \prime \prime}\left(\frac{d x}{d t}\right)^{3}+3 \theta^{\prime \prime} \frac{d x}{d t} \frac{d^{2} x}{d t^{2}}+\theta^{\prime} \frac{d^{3} x}{d t^{3}} .
\end{aligned}
$$

There result two new equations free of the time

$$
\begin{aligned}
& \left(Y-y^{\prime} X\right) y^{\prime \prime \prime}=\left|\begin{array}{cc}
1 & X_{x}+y^{\prime} X_{y}+\theta^{\prime} X_{\theta} \\
y^{\prime} & Y_{x}+y^{\prime} Y_{y}+\theta^{\prime} Y_{\theta}
\end{array}\right| y^{\prime \prime}-3 X y^{\prime \prime 2} \\
& \left(T-\theta^{\prime} X\right) \theta^{\prime \prime \prime}=\left|\begin{array}{cc}
1 & X_{x}+y^{\prime} X_{y}+\theta^{\prime} X_{\theta} \\
\theta^{\prime} & T_{x}+y^{\prime} T_{y}+\theta^{\prime} T_{\theta}
\end{array}\right| \theta^{\prime \prime}-3 X \theta^{\prime \prime 2}
\end{aligned}
$$

The quintuply infinite system of trajectorial series is completely represented by equations (9) and (10). Equation (11) is a consequence of (9) and (10). Since a trajectorial series is uniquely determined by the assignment of the initial values of $x, y, \theta, y^{\prime}, \theta^{\prime}, y^{\prime \prime}$ (this is equivalent to the assignment of the initial position and the initial velocities), the values of $\theta^{\prime \prime}$ and $y^{\prime \prime \prime}$ are given by (9) and (10) and the values of $\theta^{\prime \prime \prime}, y^{\mathrm{IV}}$, and so on, are obtained by successive differentiation of (9) and (10).

11. The differential equations of the $\infty^{5}$ trajectorial series associated with

(a) a macroscopic body,

(b) an element-particle,

(c) a microscopic body

are equations (9) and (10) where

(a) $X=X / M, Y=Y / M$, and $T=T / I$ and $X, Y, T, M, I$ are given by equations (3),

(b) $X, Y$, and $T$ are given by the right-hand members of equations (4.1), (4.2), and (4.3) respectively,

(c) $X=X / M, Y=Y / M$, and $T=T / I$ and $X, Y, T$ are given by the right-hand members of equations (6), where $M$ is the mass, and where $I$ is the polar moment of inertia about the center of gravity of the microscopic body.

The equations of THE UNIONS OF THE TRAJECTORIAL SERIES

12. To determine the equations for the unions of the trajectorial series, adjoin the conditions

$$
\theta=\arctan y^{\prime}, \quad \theta^{\prime}=\frac{y^{\prime \prime}}{1+y^{\prime 2}}, \quad \theta^{\prime \prime}=\frac{\left(1+y^{\prime 2}\right) y^{\prime \prime \prime}-2 y^{\prime} y^{\prime \prime 2}}{\left(1+y^{\prime 2}\right)^{2}}
$$


to equations (9) and (10) and eliminate $\theta$ and its derivatives. The equations (9) and (10) become

$$
\begin{aligned}
& y^{\prime \prime \prime}=P_{1} y^{\prime \prime}+Q_{1} y^{\prime \prime 2}, \\
& y^{\prime \prime \prime}=P_{2} y^{\prime \prime}+Q_{2} y^{\prime \prime 2},
\end{aligned}
$$

where

$$
\begin{aligned}
\left(\boldsymbol{Y}-y^{\prime} \boldsymbol{X}\right) P_{1} & =\left[\boldsymbol{Y}_{x}+\left(\boldsymbol{Y}_{y}-\boldsymbol{X}_{x}\right) y^{\prime}-\boldsymbol{X}_{y} y^{\prime 2}\right] \\
\left(\boldsymbol{Y}-y^{\prime} \boldsymbol{X}\right) P_{2} & =T\left(1+y^{\prime 2}\right) \\
\left(\boldsymbol{Y}-y^{\prime} \boldsymbol{X}\right) Q_{1} & =\left(\boldsymbol{Y}_{y^{\prime}}-y^{\prime} \boldsymbol{X}_{y^{\prime}}\right)-3 \boldsymbol{X} \\
\left(\boldsymbol{Y}-y^{\prime} \boldsymbol{X}\right)\left(1+y^{\prime 2}\right) Q_{2} & =-\left[\boldsymbol{X}-2 y^{\prime} \boldsymbol{Y}+3 y^{\prime 2} \boldsymbol{X}\right]
\end{aligned}
$$

13. It is seen immediately that the maximum number of trajectorial unions that can be obtained in any of the cases considered does not exceed $\infty^{3}$. The necessary and sufficient conditions that there be $\infty^{3}$ unions are

$$
P_{1} \equiv P_{2}, \quad Q_{1} \equiv Q_{2},
$$

for all $x, y, y^{\prime}$. In expanded form, these two conditions may be written

$$
\begin{aligned}
Y_{x}+\left(Y_{y}-X_{x}\right) y^{\prime}-X_{y} y^{\prime 2} & =T\left(1+y^{\prime 2}\right), \\
\left(Y_{y^{\prime}}-y^{\prime} X_{y^{\prime}}\right)\left(1+y^{\prime 2}\right) & =2\left(X+y^{\prime} Y\right) .
\end{aligned}
$$

14. For a macroscopic body $R$ with density function $\rho(\alpha, \beta)$, equations (14) and (15) are integro-differential equations on the two components of the force $\phi(x, y)$ and $\psi(x, y)$.

THEOREM 2. If $R$ is a macroscopic, radially isotropic body, there does not exist an acceleration field such that the $\infty^{5}$ trajectorial series contain $\infty^{3}$ unions.

If $R$ is radially isotropic, then $X$ and $Y$ are independent of $y^{\prime}$. The lefthand side of (15) is identically zero; the right-hand side implies that both $X$ and $Y$ are identically zero. Therefore, by equation (14), $T$ is zero. The totality of unions would be the $\infty^{2}$ straight lines of the plane.

15. Theorem 3. There does not exist an acceleration field such that the $\infty^{5}$ trajectorial series associated with an element-particle moving in the field contain $\infty^{3}$ unions.

For an element-particle, $X(=\phi)$ and $Y(=\psi)$ are independent of $y^{\prime}$; therefore by (15) both are identically zero. Equation (14) then implies that $T$ is zero. The totality of unions would be the straight lines of the plane.

TheOREM 4. There does not exist an acceleration field such that the $\infty^{5}$ trajectorial series associated with a microscopic body moving in the field contain $\infty^{3}$ unions.

For the microscopic body for which $I_{\alpha}=I_{\beta}$, the result follows as a special 
case of Theorem 2. It may be assumed, therefore, that $I_{\alpha}-I_{\beta} \neq 0$. Multiply equation (15) by $M$ and substitute into it the right-hand members of equations (6), remembering that $\tan \theta=y^{\prime}$. The resulting identity may be written as a polynomial in $y^{\prime}$ equal to zero. As each coefficient of this polynomial must be identically zero also, the following equations are implied.

$$
\begin{aligned}
M \phi+4^{-1} I \nabla^{2} \phi & =0, \\
M \psi+4^{-1} I \nabla^{2} \psi & =0, \\
\phi_{x x}-\phi_{y y}-2 \psi_{x y} & =0, \\
\psi_{x x}-\psi_{y y}+2 \phi_{x y} & =0 .
\end{aligned}
$$

In a similar manner, the equations

$$
\begin{aligned}
\left(I_{\alpha}-I_{\beta}\right)\left(\psi_{y}-\phi_{x}\right)_{x y}-2 M\left(\psi_{x}-\phi_{y}\right) & =0 \\
I\left(\phi_{x}+\psi_{y}\right)_{x y}+2 M\left(\psi_{x}+\phi_{y}\right) & =0 \\
I\left(\psi_{x}-\phi_{y}\right)_{x y}-2 M\left(\psi_{y}-\phi_{x}\right) & =0
\end{aligned}
$$

are obtained from equation (14).

Equations (16.1), (16.3), and (17.1) imply

$$
2 I_{\alpha} \phi_{y}-I \psi_{x}=0
$$

Equations (16.2), (16.4), and (18) imply

$$
I\left(I+2 I_{\alpha}\right) \psi_{x x}+4 M I_{\alpha} \psi=0
$$

equations (17.1), (17.2), and (18) imply

$$
I^{2} \psi_{x x x}+2 M\left(2 I_{\alpha}+I_{\beta}\right) \psi_{x}=0 .
$$

From (19) and (20), the equation

$$
\left(4 I_{\alpha}^{2}+3 I_{\alpha} I_{\beta}+I_{\beta}^{2}\right) \psi_{x}=0
$$

is obtained. Since $I_{\alpha} \geqq 0, I_{\beta} \geqq 0$, and $I_{\alpha}+I_{\beta}=I>0$, it must be that $\psi_{x}=0$. Equation (18) then shows that $\phi_{y}=0$ provided $I_{\alpha} \neq 0$. If $I_{\alpha}=0$, take the partial derivative with respect to $x$ of (16.4). This gives $\phi_{x x y}=0$, which, when introduced into equation (17.2), implies $\phi_{y}=0$. Thus, the only solution of equations (16) is $\phi=0$ and $\psi=0$. The theorem is proved.

16. The conditions that the $\infty^{5}$ trajectorial series contain only $\infty^{2}$ trajectorial unions are

$$
y^{\prime \prime}\left[\left(P_{1}-P_{2}\right)+\left(Q_{1}-Q_{2}\right) y^{\prime \prime}\right]=0 \text { and } y^{\prime \prime \prime}=P_{1} y^{\prime \prime}+Q_{1} y^{\prime \prime 2} \text {. }
$$

One solution is immediate: $y^{\prime \prime}=0$. Excepting the fictional "infinite rectilinear velocity," this solution is meaningful only when $X=Y=0$.

Excluding this case from further consideration, we are led to the system of equations 


$$
\begin{aligned}
&\left(Y-y^{\prime} X\right) y^{\prime \prime \prime}= {\left[Y_{x}+\left(Y_{y}-X_{x}\right) y^{\prime}-X_{y} y^{\prime 2}\right] y^{\prime \prime} } \\
&+\left[\left(Y_{y^{\prime}}-y^{\prime} X_{y^{\prime}}\right)-3 X\right] y^{\prime \prime 2} \\
& {\left[\left(Y_{y^{\prime}}-y^{\prime} X_{y^{\prime}}\right)\left(1+y^{\prime 2}\right)-2\left(X+y^{\prime} Y\right)\right] y^{\prime \prime} } \\
&=\left[T\left(1+y^{\prime 2}\right)-\left\{Y_{x}+\left(Y_{y}-X_{x}\right) y^{\prime}-X_{y} y^{\prime 2}\right\}\right]\left(1+y^{\prime 2}\right) .
\end{aligned}
$$

17. For the general element-particle,

$$
\begin{gathered}
X=\phi, \quad Y=\psi, \\
T=\frac{1}{2}\left(\psi_{x}-\phi_{y}\right)+\frac{1}{2}\left(I_{\alpha}^{*}-I_{\beta}^{*}\right)\left[\frac{\left(\psi_{x}+\phi_{y}\right)\left(1-y^{\prime 2}\right)+2\left(\psi_{y}-\phi_{x}\right) y^{\prime}}{1+y^{\prime 2}}\right]
\end{gathered}
$$

and equations (21) and (22) simplify to

$$
\begin{aligned}
\left(\psi-y^{\prime} \phi\right) y^{\prime \prime \prime} & =\left\{\psi_{x}+\left(\psi_{y}-\phi_{x}\right) y^{\prime}-\phi_{y} y^{\prime 2}\right\} y^{\prime \prime}-3 \phi y^{\prime \prime 2}, \\
2\left(\phi+y^{\prime} \psi\right) y^{\prime \prime} & =k\left\{\left(\phi_{y}+\psi_{x}\right)\left(1-y^{\prime 2}\right)+2\left(\psi_{y}-\phi_{x}\right) y^{\prime}\right\}\left(1+y^{\prime 2}\right),
\end{aligned}
$$

where $0 \leqq k=I_{\beta}^{*} \leqq 1$. If the element-particle is an element-rod, that is, if $k=0$, then (24) implies that of the $\infty^{5}$ trajectorial series associated with an element-rod moving in an arbitrary field of force, the only possible $\infty^{2}$ unions are the straight line unions. Thus if, among the $\infty^{b}$ trajectorial series associated with an element-particle moving in an arbitrary field of force, there are to be $\infty^{2}$ unions which are not straight lines, then the differential equation of these unions must be (24), where $0<k \leqq 1$. Obviously, the unions must be a subset of the curves defined by $(23)\left({ }^{4}\right)$.

18. The differential equation (24), being of specific analytic form in $y^{\prime}$ and $y^{\prime \prime}$, may be characterized geometrically in the following manner.

Through each point there passes $\infty^{1}$ unions, one union in each direction. To each union construct the osculating circle; there is one for each direction. The centers of these osculating circles lie on an equilateral hyperbola which passes through the given point. If $(x, y)$ is the fixed point, which without loss of generality may be taken as the origin, and if $(\xi, \eta)$ are the coordinates of the centers of the osculating circles, then the equation of the equilateral hyperbola is

$$
k\left(\phi_{y}+\psi_{x}\right)\left(\xi^{2}-\eta^{2}\right)+2 k\left(\psi_{y}-\phi_{x}\right) \xi \eta+2(\phi \eta-\psi \xi)=0 .
$$

19. Through the fixed point, in a fixed direction $y^{\prime}$, there passes $\infty^{1}$ of the $\infty^{8}$ curves defined by (23). Kasner $\left(^{(}\right)$has shown that if the osculating circles of these $\infty^{1}$ curves be drawn, there will be just one hyperosculating circle, and that the centers of the $\infty^{1}$ hyperosculating circles obtained by varying $y^{\prime}$ lie on a conic passing through the fixed point. In the $(\xi, \eta)$ co-

(4) The equation (23) was discovered and extensively studied by E. Kasner. Cf. Differentialgeometric aspects of dynamics, Amer. Math. Soc. Colloquium Publications, vol. 3, 1909. 424.

(\$) E. Kasner, The trajectories of dynamics, Trans. Amer. Math. Soc. vol. 7 (1906) pp. 401- 
ordinate system the equation of this conic is

$$
\phi_{y} \xi^{2}-\left(\phi_{x}-\psi_{y}\right) \xi \eta-\psi_{x} \eta^{2}+3(\phi \eta-\psi \xi)=0 .
$$

It is obvious that the equilateral hyperbola (25) and the conic (26) are tangent at the origin. Let $\left(x_{1}, y_{1}\right)$ and $\left(x_{2}, y_{2}\right)$ be the two other points of intersection. The circle through these two points and the origin is tangent to both conics at the origin. The equation of the circle, found by subtracting (26) from $(25)$, is

$$
k\left(\psi_{x}-\phi_{y}\right)\left(\xi^{2}+\eta^{2}\right)+2(1-3 k)(\phi \eta-\psi \xi)=0 .
$$

20. At the origin construct the normal common to the three conics (25), (26), and (27). If $\left(\xi_{1}, \eta_{1}\right)$ are the coordinates of the point $\omega_{1}$, which is the other intersection of the normal and the conic (25), then

$\omega_{1}: \quad \xi_{1}=\frac{2 \psi\left(\phi^{2}+\psi^{2}\right)}{k D_{1}}, \quad \eta_{1}=-\frac{2 \phi\left(\phi^{2}+\psi^{2}\right)}{k D_{1}}$,

where $D_{1}=\left(\phi_{y}+\psi_{x}\right)\left(\psi^{2}-\phi^{2}\right)-2\left(\psi_{y}-\phi_{x}\right) \phi \psi$.

Let $\left(\xi_{2}, \eta_{2}\right)$ be the coordinates of the point $\omega_{2}$, which is the other intersection of the normal and the conic (26). Then

$$
\omega_{2}: \quad \xi_{2}=\frac{3 \psi\left(\phi^{2}+\psi^{2}\right)}{D_{2}}, \quad \eta_{2}=-\frac{3 \phi\left(\phi^{2}+\psi^{2}\right)}{D_{2}},
$$

where $D_{2}=\phi_{y} \psi^{2}+\left(\phi_{x}-\psi_{y}\right) \phi \psi-\psi_{x} \phi^{2}$.

Finally let $\left(\xi_{3}, \eta_{3}\right)$ be the coordinates of the point $\omega_{3}$, which is the other intersection of the normal and the circle (27). Then

$\omega_{3}:$

$$
\xi_{3}=\frac{2(1-3 k) \psi}{k D_{3}}, \quad \eta_{3}=-\frac{2(1-3 k) \phi}{k D_{3}},
$$

where $D_{3}=\left(\psi_{x}-\phi_{y}\right)$.

It is clear that if two of the points $\omega_{i}$ are at infinity, the third point is also.

21. The significance of the points $\omega_{i}$ and the three conics is made apparent by the following theorem.

TheORem 5. If the equations (23) and (24) are compatible, then the points $\omega_{1}, \omega_{2}$, and $\omega_{3}$ are at infinity; the circle (27) has degenerated to a straight line $l_{1}$; the conic (25) to two perpendicular straight lines $l_{1}$ and $l_{2}$; and the conic (26) into two perpendicular straight lines $l_{1}$ and $l_{3}$. In the special case $k=1 / 3$, the conics (25) and (26) coincide; there is no circle (27); and the conic degenerates into two perpendicular straight lines $l_{1}$ and $l_{2}=l_{3}$.

For convenience write

$$
\gamma=2\left(\phi+v^{\prime} \psi\right) / k . \quad \lambda=\left(\psi-y^{\prime} \phi\right),
$$




$$
\begin{aligned}
& \mu=\left\{\psi_{x}+\left(\psi_{y}-\phi_{x}\right) y^{\prime}-\phi_{y} y^{\prime 2}\right\}, \\
& \delta=\mu\left(1+y^{\prime 2}\right)+\left\{\phi_{y}+\left(\psi_{y}-\phi_{x}\right) y^{\prime}-\psi_{x} y^{\prime 2}\right\}\left(1+y^{\prime 2}\right), \quad \nu=-3 \phi .
\end{aligned}
$$

Equations (23) and (24) become

$$
\begin{aligned}
\lambda y^{\prime \prime \prime} & =\mu y^{\prime \prime}+\nu y^{\prime \prime 2}, \\
\gamma y^{\prime \prime} & =\delta .
\end{aligned}
$$

The compatibility condition, equation (30), is obtained by eliminating $y^{\prime \prime}$ and $y^{\prime \prime \prime}$ between (28), (29), and the equation obtained by differentiating (29).

(30) $\gamma \delta \lambda\left(\gamma_{x}+y^{\prime} \gamma_{y}\right)+\delta^{2} \lambda \gamma_{y^{\prime}}+\gamma^{2} \delta \mu+\gamma^{2} \nu=\gamma^{2} \lambda\left(\delta_{x}+y^{\prime} \delta_{y}\right)+\gamma \delta \lambda \delta_{y^{\prime}}$.

The compatibility condition, a polynomial in $y^{\prime}$, must be identically satisfied for all values of $x, y, y^{\prime}$; and in particular, it must be satisfied at each point for $\left(^{6}\right)$

$$
y^{\prime}=-\phi / \psi \text {. }
$$

However, for this value of $y^{\prime}, \gamma=0$, so that (30) reduces to

$$
\delta^{2} \lambda \gamma_{y^{\prime}}=0 \text {. }
$$

Since $\gamma_{y^{\prime}} \equiv 2 \psi / k \neq 0$ and $\lambda$, for this value of $y^{\prime}$, is not zero, we must have $\delta=0$. In expanded form this condition is

$$
D_{1}=\left(\phi_{y}+\psi_{x}\right)\left(\psi^{2}-\phi^{2}\right)-2\left(\psi_{y}-\phi_{x}\right) \phi \psi=0,
$$

and the point $\omega_{1}$ is at infinity. Equation (32) may be interpreted to mean that $\delta$, considered as a polynomial in $y^{\prime}$, is divisible by $\gamma$, a polynomial of first degree in $y^{\prime}$. The division being performed, equation (29) becomes

$$
y^{\prime \prime}=\frac{k}{2}\left(\frac{\psi_{x}+\phi_{y}}{\phi \psi}\right)\left(\psi-y^{\prime} \phi\right)\left(1+y^{\prime 2}\right) .
$$

If there are to be $\infty^{2}$ unions different from the straight lines of the plane, it is necessary that $\left(\psi_{x}+\phi_{y}\right) \not \equiv 0$. Equation (28) may always be written in the form

$$
\frac{y^{\prime \prime \prime}}{y^{\prime \prime}}=\frac{d\left(\psi-y^{\prime} \phi\right) / d x}{\left(\psi-y^{\prime} \phi\right)}-\frac{2 \phi}{\psi-y^{\prime} \phi} y^{\prime \prime} .
$$

The compatibility condition, obtained by eliminating $y^{\prime \prime}$ and $y^{\prime \prime \prime}$ between (33), (34), and the logarithmic derivative of (33), is a polynomial of first degree in $y^{\prime}$. Equations (35) and (36) are obtained by equating each of the two

(6) It is assumed that neither $\phi$ nor $\psi$ is identically zero. If either one is zero, equation (23) is immediately integrable. Comparison of this first integral of (23) with (24) shows that the other component is also zero and that the only unions are the straight lines. 
coefficients to zero.

$$
\begin{aligned}
& \frac{\partial}{\partial x}\left(\frac{\phi \psi}{\psi_{x}+\phi_{y}}\right)=k \phi, \\
& \frac{\partial}{\partial y}\left(\frac{\phi \psi}{\psi_{x}+\phi_{y}}\right)=k \psi .
\end{aligned}
$$

The field is obviously derivable from a potential so that the point $\omega_{3}$, and consequently the point $\omega_{2}$, are at infinity. The circle (27), if $k \neq 1 / 3$, degenerates to the straight line $l_{1}$, whose equation is

$$
\phi \eta-\psi \xi=0 ;
$$

the conic (25) to the two perpendicular straight lines $l_{1}$ and $l_{2}$, where the equation of $l_{2}$ is

$$
\phi \xi+\psi \eta=\phi \psi / k \phi_{y} ;
$$

and the conic (26) to the two perpendicular straight lines $l_{1}$ and $l_{3}$, where the equation of $l_{3}$ is

$$
\phi \xi+\psi \eta=3 \phi \psi / \phi_{y} .
$$

In the special case $k=1 / 3$, the field is still derivable from a potential and the conics (25) and (26) are identical. There is no circle (27). The point $\omega_{1}$ (and $\omega_{2} \equiv \omega_{1}$ ) is at infinity by virtue of equation (32), and the conic (25) degenerates to the two perpendicular straight lines $l_{1}$ and $l_{2}=l_{3}$. This completes the proof of Theorem 5 .

22. If there are to be $\infty^{2}$ unions among the $\infty^{8}$ trajectorial series, it is necessary that the field in which the element-particle moves be potential. If the potential function $u(x, y)$ is introduced into (32), (35), and (36), these equations become

$$
\begin{gathered}
\left(u_{y y}-u_{x x}\right) u_{x} u_{y}=u_{x y}\left(u_{y}^{2}-u_{x}^{2}\right), \\
\frac{\partial}{\partial x}\left(\frac{u_{x} u_{y}}{u_{x y}}-2 k u\right)=0, \\
\frac{\partial}{\partial y}\left(\frac{u_{x} u_{y}}{u_{x y}}-2 k u\right)=0 .
\end{gathered}
$$

Equations (38) and (39) imply

$$
\frac{u_{x} u_{y}}{u_{x y}}-2 k u=c .
$$

Obviously, if $u$ is a solution of (40) for $c=0$, then $(u-c / 2 k)$ is a solution of (40) for $c \neq 0$. Since $u$ is a potential function and only its derivatives are 
needed, we may, therefore, without loss of generality, set $c=0$. The functions $u(x, y)$ which satisfy $(40)$ when $c=0$ separate into two classes which are distinguished by the symmetry or asymmetry of the element-particle. If the element-particle is symmetric, that is, if $k=1 / 2$, then

$$
u=e^{\sigma_{1}+\sigma_{2}}
$$

where $\sigma_{1}$ is a function of $x$ alone and $\sigma_{2}$ is a function of $y$ alone. If, however, the element-particle is asymmetric, that is, if $k \neq 1 / 2$, then

$$
u=\left(\sigma_{1}+\sigma_{2}\right)^{2 k /(2 k-1)} \text {. }
$$

The substitution of either (41) or (42) into (37) leads, after the suppression of the obvious nonzero factors, to the equation

$$
\frac{d \sigma_{1}}{d x} \frac{d \sigma_{2}}{d y}\left(\frac{d^{2} \sigma_{1}}{d x^{2}}-\frac{d^{2} \sigma_{2}}{d y^{2}}\right)=0 .
$$

Neither of the first two factors of (43) is zero. If either were zero, $u$ would be a function of only one variable; therefore, one of the components of the field of force would be zero. However, we have seen that if one component is zero the compatibility condition requires that the other also be zero; therefore, the only unions would be the straight lines. Thus, only the third factor is zero and

$$
\frac{d^{2} \sigma_{1}}{d x^{2}}=\frac{d^{2} \sigma_{2}}{d y^{2}}=2 a_{1}
$$

since $\sigma_{1}$ is a function of $x$ alone and $\sigma_{2}$ of $y$ alone. Integrating, we obtain

$$
\sigma_{1}+\sigma_{2}=a_{1}\left(x^{2}+y^{2}\right)+2 a_{2} x+2 a_{3} y+a_{4} \text {. }
$$

The differential equation (33) may be written, after the introduction of the function $2 G=\log u$, as follows: $y^{\prime \prime}=\left(G_{y}-y^{\prime} G_{x}\right)\left(1+y^{\prime 2}\right)$.

THEOREM 6. If a symmetric element-particle moves in such a field of force that there are $\infty^{2}$ non-straight line unions among the $\infty^{5}$ trajectorial series, then the potential function $u$ for the field is given by (41) and (44) and the differential equation of these $\infty^{2}$ unions is

$$
y^{\prime \prime}=2\left[\left(a_{1} y+a_{3}\right)-y^{\prime}\left(a_{1} x+a_{2}\right)\right]\left(1+y^{\prime 2}\right) .
$$

THEOREM 7. If an asymmetric element-particle moves in such a field of force that there are $\infty^{2}$ non-straight line unions among the $\infty^{5}$ trajectorial series, then the potential function $u$ for the field is given by (42) and (44) and the differential equation of these $\infty^{2}$ unions is

$$
y^{\prime \prime}=\left(\frac{4 k}{2 k-1}\right)\left[\frac{\left(a_{1} y+a_{3}\right)-y^{\prime}\left(a_{1} x+a_{2}\right)}{a_{1}\left(x^{2}+y^{2}\right)+2 a_{2} x+2 a_{3} y+a_{4}}\right]\left(1+y^{\prime 2}\right) .
$$




\section{Geometric CHARACTERIZATION OF THE UNIONS}

23. The object of this section is to provide a complete geometric characterization of the equations of the trajectorial unions, equations (45) and (46). To simplify the discussion, we apply a translation to the $(x, y)$ plane and write these equations as follows

$$
\begin{aligned}
& y^{\prime \prime}=a\left(y-y^{\prime} x\right)\left(1+y^{\prime 2}\right) \\
& y^{\prime \prime}=\left(\frac{4 k}{2 k-1}\right) \frac{\left(y-y^{\prime} x\right)\left(1+y^{\prime 2}\right)}{\left(x^{2}+y^{2}+b\right)} .
\end{aligned}
$$

24. Property I. A set of $\infty^{2}$ curves of the plane is a velocity system if the centers of the $\infty^{1}$ osculating circles of the $\infty^{1}$ curves of the set through the point $(x, y)$, constructed at $(x, y)$, lie on a straight line $\left.{ }^{7}\right)$.

THEOREM 8. The set of $\infty^{2}$ trajectorial unions is a velocity system.

For the symmetric particle, the centers of the $\infty^{1}$ osculating circles, constructed at the point $(x, y)$, of the $\infty^{1}$ curves of the set through $(x, y)$ lie on the straight line whose equation, in the $(\xi, \eta)$ coordinate system, is

$$
a(x \xi+y \eta)=a\left(x^{2}+y^{2}\right)+1 ;
$$

whereas, for the asymmetric particle, the centers of the osculating circles lie on the straight line whose equation is

$$
4 k(x \xi+y \eta)=(6 k-1)\left(x^{2}+y^{2}\right)+(2 k-1) b .
$$

25. To each point $p$ there corresponds, by Property I, a line $l_{p}$; to each point $p$ and line $l_{p}$ there corresponds a line $l_{p}^{\prime}$, perpendicular to $l_{p}$ and passing through $p$.

\section{Theorem 9. Property II. The totality of lines $l_{p}^{\prime}$ form a pencil.}

The equation of a line perpendicular to (49) or (50) and passing through the point $(x, y)$ is

$$
y \xi-x \eta=0 .
$$

26. It is obvious also that if two points $p$ and $\bar{p}$ are on the same line $l^{\prime}$, then the two lines $l_{p}$ and $l_{\bar{p}}$ are parallel. Under what conditions will these two lines coincide?

For the symmetric element-particle, the equation of the line $l_{p}$ is (49) and the equation of the line $l_{\bar{p}}$ is (49) with $x$ replaced by $\bar{x}$ and $y$ by $\bar{y}$. If these two equations are to represent the same line, the coefficients must be proportional. It is an easy consequence of the proportionality equations that

$\left(^{7}\right)$ E. Kasner and J. DeCicco, The geometry of velocity systems, Bull. Amer. Math. Soc. vol. 49 (1943) pp. 236-245. 


$$
\bar{x}=\frac{x}{a\left(x^{2}+y^{2}\right)}, \quad \bar{y}=\frac{y}{a\left(x^{2}+y^{2}\right)},
$$

that is, $p$ and $p$ are inversive in a circle of $\operatorname{radius}\left({ }^{8}\right)(1 / a)^{1 / 2}$, a constant for a given field, centered at the origin.

Theorem 10. Property III $_{s}$ (for the symmetric particle). To each point $p$ there corresponds a point $\bar{p}$, which is inversive to $p$ in a circle of fixed radius $\left({ }^{8}\right)$ about the origin, such that the centers of the $\infty^{1}$ osculating circles constructed at $p$ lie on the same straight line as the centers of the $\infty^{1}$ osculating circles constructed at $p$.

For the asymmetric element-particle the situation is complicated by special cases. In the "general" case, for which $(6 k-1) \neq 0$ and $b \neq 0$, Property III is applicable. The relation between $p$ and $p$ is

$$
\bar{x}=b\left(\frac{2 k-1}{6 k-1}\right) \frac{x}{x^{2}+y^{2}}, \quad \bar{y}=b\left(\frac{2 k-1}{6 k-1}\right) \frac{y}{x^{2}+y^{2}} .
$$

If either or both $(6 k-1)=0, b=0$, we do not consider a Property III.

27. For each point $p$ there are defined two lines $l_{p}$ and $l_{p}^{\prime}$. The point of intersection of these two lines is called $p^{*}$. Let the distance from the center of the pencil of lines $l_{p}^{\prime}$ to the point $p$ be $s$, to the point $\bar{p}$ be $\bar{s}$, and to the point $p^{*}$ be $s^{*}$.

Theorem 11. Property $\mathrm{IV}_{s}$ (the symmetric case).

$$
s^{*}=s+\bar{s} \text {. }
$$

If $\left(x^{*}, y^{*}\right)$ are the coordinates of $p^{*}$, then

$$
x^{*}=x+\frac{x}{a\left(x^{2}+y^{2}\right)}=x+\bar{x} ;
$$

similarly for $y^{*}$; and the conclusion is obvious.

Theorem 12. Property $\mathrm{IV}_{a}$. For the general asymmetric case there exists a constant $h \neq 0,1,-\infty<h \leqq 5 / 4$, such that

$$
s^{*}=h(s+\bar{s}) \text {. }
$$

If $\left(x^{*}, y^{*}\right)$ are the coordinates of the point $p^{*}$, then

$$
x^{*}=h\left[x+b\left(\frac{2 k-1}{6 k-1}\right) \frac{x}{x^{2}+y^{2}}\right]=h(x+\bar{x})
$$

where $h=(6 k-1) / 4 k$; similarly for $y^{*}$; and the conclusion is obvious.

TheOREM 13. Property IV IV $^{\prime}$. In the special asymmetric case for which $k=1 / 6$

$\left({ }^{8}\right)$ The radius may be imaginary. 
and $b \neq 0$, the points $p$ and $p^{*}$ are inversive in a circle of fixed radius, center at the origin.

If $\left(x^{*}, y^{*}\right)$ are the coordinates of the point $p^{*}$, then

$$
x^{*}=-b \frac{x}{x^{2}+y^{2}}=h \frac{x}{x^{2}+y^{2}}
$$

and similarly for $y^{*}$.

ThEOREM 14. Property IV $_{a}^{\prime \prime}$. In the special asymmetric case for which $(6 k-1) \neq 0$ and $b=0$, there exists a constant $h \neq 0,1,-\infty<h \leqq 5 / 4$, such that

$$
s^{*}=h s \text {. }
$$

In particular, $h=(6 k-1) / 4 k$.

THEOREM 15. Property IV IV $^{\prime \prime}$. In the special asymmetric case for which $k=1 / 6$ and $b=0$, the lines $l_{p}$ form $a$ pencil identical with the pencil of lines $l_{p}^{\prime}$.

For $k=1 / 6$ and $b=0$, equation (50) becomes

$$
x \xi+y \eta=0,
$$

which is the equation of a pencil of lines identical with the pencil of lines defined by equation (51).

\section{Converse questions}

28. This section is concerned with the inquiry into the extent the geometric properties obtained for the trajectorial unions are characteristic of such systems. If a two-parameter family of curves has the set of properties $\mathrm{I}, \mathrm{II}, \mathrm{III}_{\mathrm{s}}, \mathrm{IV}_{\mathrm{s}}$, or

$\mathrm{I}, \mathrm{II}, \mathrm{III}_{a}, \mathrm{IV}_{a}$, or

I, II, IV'

I, II, IV ${ }_{a}^{\prime \prime}$, or

$$
\text { I, II, IV }{ }_{a}^{\prime \prime \prime}
$$

is it a family of trajectorial unions? It will be shown that the answer is yes.

29. Given that the two-parameter family of curves defined by the differential equation

$$
y^{\prime \prime}=g\left(x, y, y^{\prime}\right)
$$

has Property I, that is, that the set of $\infty^{2}$ curves is a velocity system, and that the equation of the line $l_{p}$, on which lie the centers of the $\infty^{1}$ osculating circles constructed at the point $(x, y)$ to the $\infty^{1}$ curves of the set passing 
through $(x, y)$, is

$$
A_{0}(\xi-x)+B_{0}(\eta-y)=1
$$

where $A_{0}$ and $B_{0}$ are arbitrary functions of $(x, y)$, then equation (52) has the form

$$
y^{\prime \prime}=\left(B_{0}-y^{\prime} A_{0}\right)\left(1+y^{\prime 2}\right) .
$$

30. If a set of $\infty^{2}$ curves has, in addition to Property I, Property II, then the functions $A_{0}$ and $B_{0}$ are not arbitrary, but satisfy some specific condition. Property II states, in effect, that there exists a fixed point, which for convenience is taken as the origin, such that the line $l_{p}^{\prime}$, which is perpendicular to the line $l_{p}$ whose equation is (53) and passes through the point $(x, y)$, passes through the fixed point, the origin. The equation of the line $l_{p}^{\prime}$ is

$$
B_{0}(\xi-x)-A_{0}(\eta-y)=0 .
$$

As this line is to contain the fixed point, the origin, we must have

$$
B_{0} x-A_{0} y=0 \text {. }
$$

Therefore, the differential equation of any two-parameter family of curves which has Properties I and II is

$$
y^{\prime \prime}=A_{1}\left(y-y^{\prime} x\right)\left(1+y^{\prime 2}\right)
$$

where $A_{1}=A_{0} / x$ is an arbitrary function of $x$ and $y$.

31. The set $\Sigma_{5}$.

THEOREM 16. The equation of the family of $\infty^{2}$ curves which has all of the properties of the set $\Sigma_{5}$ is obtained by setting $k=1 / 6$ and $b=0$ in equation (48).

To prove this, note that Property $\mathrm{IV}_{a}^{\prime \prime \prime}$ implies that the line $l_{p}$, whose equation is (53), passes through the origin. Setting $\xi, \eta$ equal to zero and, with (55), solving for $A_{0}$, we find that

$$
A_{0}=-\frac{x}{x^{2}+y^{2}}
$$

Thus, (57) becomes

$$
y^{\prime \prime}=-\frac{\left(y-y^{\prime} x\right)\left(1+y^{\prime 2}\right)}{\left(x^{2}+y^{2}\right)}
$$

which is, of course, identical with the specialized equation (48).

32. The set $\Sigma_{4}$.

THEOREM 17. The equation of the two-parameter set of curves defined by the set of properties $\Sigma_{4}$ is obtained by setting $b=0$ in equation (48). 
The coordinates of the point $p^{*}$, the intersection of the lines whose equations are (53) and (55), are

$$
x^{*}=x+\frac{x}{A_{1}\left(x^{2}+y^{2}\right)}, \quad y^{*}=y+\frac{y}{A_{1}\left(x^{2}+y^{2}\right)} .
$$

Property $\mathrm{IV}_{a}^{\prime \prime}$ states that $s^{*}=h s$, where $h \neq 0,1,-\infty<h \leqq 5 / 4$. However, since $p$ and $p^{*}$ are on the same line through the origin, we may infer $x^{*}=h x$. $A_{1}$ is easily determined and when substituted in (57) reduces that equation to

$$
y^{\prime \prime}=\left(\frac{1}{h-1}\right) \frac{\left(y-y^{\prime} x\right)\left(1+y^{\prime 2}\right)}{\left(x^{2}+y^{2}\right)}
$$

which is identical with (48) when $b=0$ and $h=(6 k-1) / 4 k$.

33. The set $\Sigma_{3}$.

THEOREM 18. The differential equation of the curves defined by the set of properties $\Sigma_{3}$ is obtained by setting $k=1 / 6$ in equation (48).

Property $I_{a}^{\prime}$ states that $p$ and $p^{*}$ are inversive in a circle of fixed radius $h$, center at the origin. Thus, from (58),

$$
A_{1}=-\left(x^{2}+y^{2}-h\right)^{-1}
$$

and, when substituted in (57), reduces that equation to

$$
y^{\prime \prime}=-\frac{\left(y-y^{\prime} x\right)\left(1+y^{\prime 2}\right)}{\left(x^{2}+y^{2}-h\right)}
$$

which is identical with (48), provided $k=1 / 6$ and we identify $b$ with $-h$.

This concludes the sufficiency of the geometric characterization of the "special" asymmetric cases; there remain the symmetric and general asymmetric cases.

34. Property III. We have seen that the differential equation of any twoparameter family of curves which has Properties I and II is of the form (57). The centers of the $\infty^{1}$ osculating circles constructed, at the point $p$, to the $\infty^{1}$ curves of the family passing through $p$ lie on the straight line $l_{p}$, whose equation is

$$
A_{1}[x(\xi-x)+y(\eta-y)]=1 .
$$

We define the function

$$
A_{2} \equiv-\left[(m-1)\left(x^{2}+y^{2}\right)+m n-1 / A_{1}\right]
$$

where $m$ and $n$ are constants which will be determined later, so that we may write (59) as follows

$$
x \xi+y \eta=m\left(x^{2}+y^{2}\right)+m n+A_{2} .
$$


By Property III, there exists a circle, center at the origin and of radius $n$, such that to every pair of points $p$ and $p$, which are inverse points in this fixed circle, there corresponds the same line $l_{p}$. The equation of the line $l_{p}$ associated with the point $p$ is (60); the equation of the line $l_{\bar{p}}$ associated with the point $\bar{p}$ is

$$
\bar{x} \xi+\bar{y} \eta=m\left(\bar{x}^{2}+\bar{y}^{2}\right)+m n+\bar{A}_{2},
$$

where $\bar{A}_{2}$ is obtained by replacing $x$ and $y$ by $\bar{x}$ and $\bar{y}$ respectively in $A_{2}$. Since $p$ and $\bar{p}$ are inverse points in the circle of radius $n$, equations (60) and (61) represent the same straight line; therefore, we must have

$$
A_{2}\left(\frac{n x}{x^{2}+y^{2}}, \frac{n y}{x^{2}+y^{2}}\right) \equiv \frac{n}{x^{2}+y^{2}} A_{2}(x, y) .
$$

The only functions $A_{2}$ which satisfy (62) are of the type

$$
A_{2}=x f(y / x),
$$

where $f$ is an arbitrary function of the ratio $y / x$. To prove this, one need but take only the partial derivative of (62) with respect to $x$, obtain the linear partial differential equation for homogeneous functions of order one

$$
x \frac{\partial A_{2}}{\partial x}+y \frac{\partial A_{2}}{\partial y}=A_{2}
$$

and integrate.

Thus far it has been proved that the differential equation of any twoparameter family of curves which has Properties I, II, and III is of the form

$$
y^{\prime \prime}=\frac{\left(y-y^{\prime} x\right)\left(1+y^{\prime 2}\right)}{\left[(m-1)\left(x^{2}+y^{2}\right)+m n+x f(y / x)\right]} .
$$

35. The set $\Sigma_{2}$. Property $\mathrm{IV}_{a}$ states that there exists a constant $h \neq 0,1$, $-\infty<h \leqq 5 / 4$, such that

$$
s^{*}=h(s+\bar{s}) \text {. }
$$

However, since the points $p, \bar{p}$, and $p^{*}$ are on the same line $l_{p}^{\prime}$, this relation may be replaced by

$$
x^{*}=h(x+\bar{x})=h\left(x+\frac{n x}{x^{2}+y^{2}}\right) .
$$

The coordinate $x^{*}$ may be found also by eliminating $y^{*}$ between the equations of $l_{p}$ and $l_{p}^{\prime}$. When determined in this manner,

$$
x^{*}=m x+\frac{m n x}{x^{2}+y^{2}}+\frac{x^{2}}{x^{2}+y^{2}} f(y / x) .
$$


Thus,

$$
\frac{f(z)}{1+z^{2}} \equiv(h-m) x\left[1+\frac{n}{x^{2}\left(1+z^{2}\right)}\right],
$$

where $y / x$ has been replaced by $z$. Since $x$ and $z$ are independent variables and (64) is to be true for all values of $x$ and $z$, we conclude that $f \equiv 0$ and $m=h$.

THEOREM 19. The equation of the two-parameter family of curves defined by the set of properties $\Sigma_{2}$ is

$$
y^{\prime \prime}=\frac{\left(y-y^{\prime} x\right)\left(1+y^{\prime 2}\right)}{(h-1)\left(x^{2}+y^{2}\right)+h n},
$$

which is identical with (48), provided $h=(6 k-1) / 4 k$ and $n=b(2 k-1) /(6 k-1)$.

36. The set $\Sigma_{1}$. The procedure of the preceding article, at least so far as equation (64), remains valid in this case. Therefore, we must have again $f \equiv 0$ and $m=1$.

THEOREM 20. The equation of the two-parameter family of curves defined by the set of properties $\Sigma_{1}$ is

$$
y^{\prime \prime}=\frac{1}{n}\left(y-y^{\prime} x\right)\left(1+y^{\prime 2}\right),
$$

which is identical with (47) provided that $n=1 / a$.

37. It will be remembered that the most general differential equation of the $\infty^{2}$ trajectorial unions is either (45) for the symmetric case or (46) for the asymmetric case; the equations (47) and (48) are only special cases. In order to restate all of the geometric properties associated with a system of trajectorial unions given by equations (45) or (46), one need require only that the vertex of the pencil of lines $l_{p}^{\prime}$ (Property II) be at the point $\left(-a_{2} / a_{1}\right.$, $\left.-a_{3} / a_{1}\right)$ instead of at the origin.

THEOREM 21. The necessary and sufficient conditions that a two-parameter family of curves be trajectorial unions are that it has one of the sets of properties $\Sigma_{1}, \Sigma_{2}, \Sigma_{3}, \Sigma_{4}, \Sigma_{5}$.

38. Since the equations (47) and (48) are invariant under a rotation about the origin, it may be shown quite simply that both may be integrated by quadratures. It follows, of course, that equations (45) and (46) may be integrated also by quadratures.

Columbia University,

NEw York, N. Y. 